
\section{$C_{\text {citus }}$}

Oxford Centre of Respirato
Medicine, The Churchill

Hospital, Oxford University

Hospitals NHS Trust,

Headington, Oxford, UK

${ }^{2}$ Department of Respiratory

Medicine, Royal United

Hospital, Bath, UK

${ }^{3}$ Department of Clinical and

Experimental Sciences

University of Southampton,

Southampton, UK

\section{Correspondence to}

Dr F Maxine Hardinge, Oxford

Centre of Respiratory Medicine

The Churchill Hospital,

Oxford University Hospitals

NHS Trust, Headington,

Oxford OX3 7LJ, UK:

maxine.hardinge@ouh.nhs.uk

Received 10 February 2015

Revised 24 March 2015

Accepted 27 March 2015

Published Online First

27 April 2015

\title{
Guideline update: The British Thoracic Society Guidelines on home oxygen use in adults
}

\author{
Maxine Hardinge, ${ }_{1}^{1}$ Jay Suntharalingam, ${ }^{2}$ Tom Wilkinson $^{3}$
}

ABSTRACT

The 2015 British Thoracic Society (BTS) Home Oxygen Guidelines provides detailed evidence-based guidance for the use of oxygen by patients in their own homes or other non-acute hospital settings.

The new BTS Home Oxygen Guidelines published in this edition of Thorax provides detailed, evidence-based guidance for the use of oxygen by patients in their own homes or other non-acute hospital settings, and offers practical advice where evidence is absent or inconclusive. Its scope extends to patients with a variety of long-term respiratory conditions and also other patient groups currently prescribed home oxygen, such as those with cardiac failure and cluster headaches.

The guideline explores the evidence base for use of different modalities of oxygen and patient related outcomes such as effects on mortality, symptoms and quality of life. In doing this, it addresses current clinical controversies such as oxygen prescription to smokers, provision of oxygen on discharge from hospital, and use of palliative oxygen to support patients at the end of life. The guideline aims to support clinical decision making around patient selection, assessment and equipment choice and offers a guide to follow-up, including oxygen withdrawal. It includes a safety section where we have addressed concerns about fire risks and outlined a practical approach to risk assessment.

Home oxygen is widely prescribed, but often without rigorous patient assessment or plans in place for follow-up, leading to a recognised waste of resources. In writing the guidelines, we were aware that public perception is often that oxygen therapy is a good treatment for breathlessness rather than a treatment for hypoxaemia and, so, a focus on increased awareness and education is important.

The guide is written for use by all healthcare professionals involved in the assessment, prescription and review of home oxygen therapy. The target audience therefore consists of specialised home oxygen review services in addition to general practitioners (GPs) and practice nurses, palliative care teams, and community respiratory and pulmonary rehabilitation teams. We hope that hospital specialist teams arranging home oxygen for patients about to be discharged from acute general medicine, respiratory medicine, cardiology, neurology, oncology, and geratology wards will also find key information.
The Guideline Development Group comprised a range of practising healthcare professionals actively involved in the care of a wide variety of home oxygen patients, and importantly patient representatives themselves. The group has tackled existing problems around the use of terminology and definitions for different types of oxygen therapy. After much discussion, we adopted a pragmatic approach and we hope that clearer definitions of some existing terms, along with newer categories of home oxygen might bring greater clarity. We have settled on the use of the terms, long term, ambulatory, short burst, nocturnal and palliative oxygen therapy, although whether their associated abbreviations-LTOT, AOT, SBOT, NOT and POTprove popular remains to be seen.

Certain modalities of home oxygen, such as short burst oxygen, are recognised to have a poor evidence base to support their use. Despite this fact, and existing guidance, high levels of SBOT have been regularly provided for patients, at a significant cost to the health service suggesting that clearer evidence-based guidance is needed. The previous clinical guidelines from the Royal College of Physicians, London (RCP), in 1999, ${ }^{1}$ were updated in the "NHS Primary Care Commissioning Oxygen Service Assessment and Review Good practice guide" in 2011. This provided both advice and case studies for the increasing number of Home Oxygen Assessment and Review services that were being commissioned at that time. In parallel with this, there were changes made to the nationally awarded contracts for home oxygen providers, leading to more cost-efficient and safety-aware services. The new BTS guidelines complements the existing good practice guide by providing the evidence base for home oxygen use, and sits alongside the BTS guidance on home oxygen in children and the BTS Guidelines for Emergency Oxygen Use in Adult Patients (http://www.brit-thoracic.org.uk).

LTOT, that is, oxygen administered for at least $15 \mathrm{~h}$ per day in chronically hypoxaemic patients, remains the most evidence-based form of domiciliary oxygen in current use. The evidence for LTOT continues to be centred on two landmark randomised controlled trials (RCTs) carried out in the early 1980s that showed a survival benefit in COPD patients. ${ }^{2} 3$ The robust nature of this data allowed the Guideline Development Group to propose Grade A recommendations for this patient cohort, which are unlikely to be challenged by new evidence in the foreseeable future. Unfortunately, such robust data were lacking in other disease groups such as interstitial lung disease, cystic 
fibrosis, pulmonary hypertension or advanced cardiac failure. In these patient groups, the Guideline Development Group felt it was reasonable to extrapolate from COPD data, albeit with Grade D recommendations, on the presumption that end-organ damage from resting hypoxaemia was universally harmful regardless of the type of lung pathophysiology that had caused it. Given the evidence supporting the use of LTOT in hypoxic patients, it is important that potential LTOT candidates are screened and referred in a timely fashion. The guidelines also provide recommendations on this aspect, balancing the need to identify such patients early against the potential for overprescribing LTOT to 'unstable' patients who have failed to recover sufficiently from an exacerbation. Pragmatic advice is also given on how to manage the patient who remains profoundly hypoxic at the time of discharge from hospital.

Many discussions were held on the assessment process for oxygen. Here the Guideline Development Group advocates the use of arterial blood gases (ABGs) over capillary blood gases (CBGs) as the evidence suggests that using CBGs to select patients may lead to patients being treated with LTOT unnecessarily. ${ }^{4}$ This may have resource and training implications that individual home oxygen services will have to consider along with factors such as patient preference. However, the Guideline Development Group felt that guideline methodology mandated recommending the evidence-based approach and also highlighted that further studies are needed comparing the two techniques.

Assessment was not the only area where a lack of evidence impacted the advice given by the guide. This was particularly apparent during the discussions around use of AOT in patients who do not fulfil the criteria for LTOT, where the evidence for benefit is weak. A number of single assessment cross-over studies carried out under carefully controlled conditions suggest that AOT may improve physiological parameters in the short term. ${ }^{5}$ However, longer term clinical trials suggest that under 'real life' conditions, AOT does not confer substantial long-term benefits and is frequently underutilised by patients. ${ }^{6}$ On balance it was therefore felt there was insufficient data to support routine use of AOT in non-LTOT patients. As with LTOT, there was limited data in non-COPD patient groups and thus recommendations had to be based on extrapolation, despite the fact that pathophysiology, and hence propensity to de-saturate, can vary widely between respiratory conditions. Again, these limitations in the existing evidence base highlight the need for further research in the future.

The new guidelines offer insights into and advice on the safety of prescribing home oxygen, recognising that in common with any prescribed therapy, oxygen has the potential to cause harm to the individual; but unlike many therapies, oxygen also has the potential, due to its flammable nature, to cause great harm to carers, co-habitants and others. The complexity of decision making around this issue is increased by the additional factor that cigarette smoking remains prevalent in patients with advanced lung disease. This guideline has a new section on the safety concerns and considerations for the prescription of oxygen therapy in each individual's case and offers practical advice to the practitioner for the assessment, estimation and mitigation of risk. The need to assess the home environment including risks of trips and falls associated with oxygen equipment, adequately assess the smoking status and offer written advice to patients and carers, highlighting risks are recommended. While the Guideline Development Group felt it is important that the responsible clinician is left with the final decision regarding oxygen prescription, we hope that the

\section{Box 1 Key recommendations}

1. Long-term oxygen therapy (LTOT) remains the most evidence-based form of home oxygen therapy, with no change to current indications and thresholds.

2. Ambulatory oxygen therapy assessment should only be offered to patients already on LTOT if they are mobile outdoors.

3. Treatment with palliative oxygen therapy does not have a role in patients with cancer or end-stage cardio-respiratory disease with intractable breathlessness if their resting oxygen levels are normal or only mildly hypoxaemic but above current LTOT thresholds.

4. Short burst oxygen therapy should not be ordered for use prior to or following exercise in chronic obstructive pulmonary disease patients whether or not they have resting hypoxaemia.

5. A risk assessment approach should be adopted for assessing safety of all forms of home oxygen especially in smokers

guidelines include an informed appraisal of both clinical indication and the potential risk of therapy.

The process of producing evidence-based guidelines such as this is now a rigorous and highly standardised one. The Guideline Development Group was able to review the entire literature available in the English language in this area and, while this was a significant task, it became apparent that in many, if not most, areas there was a distinct lack of high quality evidence

\section{Box 2 Research recommendations}

1. Investigation of which patients with particular disease phenotypes benefit from long-term oxygen therapy (LTOT): for example, smokers compared with ex-smokers, those with pulmonary hypertension, those with chronic obstructive pulmonary disease (COPD) driven cachexia and frequent exacerbators.

2. Investigation of long-term outcomes (survival) with LTOT therapy in diseases other than COPD such as cystic fibrosis, interstitial lung disease and bronchiectasis.

3. Investigation of delivery of oxygen during pulmonary rehabilitation and maintenance classes, assessing the impact on outcomes such as exacerbations, exercise tolerance and quality of life.

4. Longitudinal studies to assess the impact of LTOT on pulmonary artery pressures using echocardiogram outcomes in COPD patients with pulmonary hypertension, along with quality of life and exercise tolerance outcomes.

5. A robust assessment of risk assessment measures with the aim of developing an integrated pathway for home oxygen teams and oxygen provider services to manage patients who smoke.

6. Investigation of the role of palliative oxygen in comparison with or used together with other measures such as opiates, fan therapy and cognitive behavioural therapy.

7. Comparison of use of arterial blood gases and capillary blood gases in predicting the need for LTOT and risk of hypercapnia. 
to inform practice and support clinical pathway design and delivery. Where evidence does exist, many of the recommendations in the 2015 guidelines rely on clinical trials performed many decades ago. The findings of these key studies, while being important in an evidence-poor environment, may be less relevant today, as other therapies for the conditions concerned have evolved considerably in recent years. Therefore, the Guideline Development Group felt that it was important to highlight both our key recommendations (box 1) and also the areas of research required to improve the evidence base for home oxygen therapy in adults (box 2). Addressing these questions will improve our understanding of the rationale for assessment, prescription, administration and impact of oxygen in the domiciliary environment, and increase the benefits and value of this important and expensive intervention.

Contributors $\mathrm{MH}$ is the lead author, and chair of the BTS home oxygen guideline group, and has overall responsibility for the content of the article. MH, JS and TW all contributed to the development, drafting and final approval of the article.
Competing interests None declared.

Provenance and peer review Not commissioned; internally peer reviewed.

\section{REFERENCES}

1 Wedzicha JA. Domiciliary oxygen therapy services: clinical guidelines and advice for prescribers. Summary of a report of the Royal College of Physicians. J $R$ Coll Physicians Lond 1999;33:445-7.

2 [No authors listed]. Continuous or nocturnal oxygen therapy in hypoxemic chronic obstructive lung disease: a clinical trial. Nocturnal Oxygen Therapy Trial Group. Ann Int Med 1980;93:391-8.

3 [No authors listed]. Long term domiciliary oxygen therapy in chronic hypoxic cor pulmonale complicating chronic bronchitis and emphysema. Report of the Medical Research Council Working Party. Lancet 1981;1:681-6.

4 Eaton T, Rudkin S, Garrett JE. The clinical utility of arterialized earlobe capillary blood in the assessment of patients for long-term oxygen therapy. Respir Med 2001;95:655-60.

5 Bradley JM, O'Neill B. Short term ambulatory oxygen for chronic obstructive pulmonary disease. Cochrane Database Syst Rev 2005;(4):CD004356.

6 Moore RP, Berlowitz DJ, Denehy L, et al. A randomised trial of domiciliary, ambulatory oxygen in patients with COPD and dyspnoea but without resting hypoxaemia. Thorax 2011;66:32-7. 